\title{
Discovery of seven ZZ Ceti stars using a new photometric selection method ${ }^{\star}$
}

\author{
B. Voss ${ }^{1}$, D. Koester ${ }^{1}$, R. Østensen ${ }^{2}$, S. O. Kepler ${ }^{3}$, R. Napiwotzki ${ }^{4}$, D. Homeier ${ }^{5}$, and D. Reimers ${ }^{6}$ \\ 1 Institut für Theoretische Physik und Astrophysik, Universität Kiel, Leibnizstraße 15, 24098 Kiel, Germany \\ e-mail: voss@astrophysik.uni-kiel.de \\ 2 Isaac Newton Group, Apartado de correos 321, 38700 Santa Cruz de la Palma, Spain \\ 3 Instituto de Física, Universidade Federal do Rio Grande do Sul, 91501-900 Porto Alegre, RS, Brazil \\ ${ }^{4}$ Centre for Astrophysics Research, University of Hertfordshire, College Lane, Hatfield AL10 9AB, UK \\ 5 Institut für Astrophysik, Georg-August-Universität, Friedrich-Hund-Platz 1, 37077 Göttingen, Germany \\ ${ }^{6}$ Hamburger Sternwarte, Gojenbergsweg 112, 21029 Hamburg, Germany
}

Received 19 September 2005 / Accepted 28 November 2005

\section{ABSTRACT}

We report the detection of non-radial $g$-mode oscillations in seven relatively bright $(14.4<B<16.5)$ DA white dwarf stars from time-series photometry collected at the Nordic Optical Telescope (NOT) at Roque de Los Muchachos Observatory, Spain. The candidate variable stars were selected from preparatory photometric observations of objects from the Hamburg Quasar Survey (HQS) and from spectroscopy of stars from the Villanova (WD) catalogue. The selection of ZZ Ceti candidate stars from photometric data proved nearly as successful as the more common spectroscopic selection method. The effective temperatures that we derive from both methods are consistent with a pure ZZ Ceti instability strip.

Key words. stars: white dwarfs - stars: oscillations - stars: variables: general

\section{Introduction}

ZZ Ceti stars or variable DA (DAV) stars are white dwarf (WD) stars with a hydrogen atmosphere that show non-radial $g$-mode oscillations. These oscillations, with known periods from $71 \mathrm{~s}$ (G 185-32, Kepler et al. 2000) up to 1466 s (HS 0952+1816, Mukadam et al. 2004a), can be detected as multi-periodic luminosity variations with amplitudes of the pulsation modes ranging from a fraction of a percent up to about five percent.

ZZ Ceti white dwarfs are found in an instability strip between effective temperatures of $\sim 10700 \mathrm{~K}$ and $\sim 12100 \mathrm{~K}$, for a typical surface gravity of $\log g=8.0$ (Bergeron et al. 2004; Mukadam et al. 2004b). Pulsators near the cool edge of the strip, referred to as cDAV stars, generally show longer periods with higher amplitudes than their hot edge counterparts, called hDAV stars.

Through seismological analysis, DAV pulsations can be employed to reveal characteristics of the interior structure of

^ Based on observations made with the Nordic Optical Telescope, and partially based on observations collected at the Centro Astronómico Hispano Alemán (CAHA) at Calar Alto, as well as on data obtained at the Paranal Observatory of the European Southern Observatory for programmes 165.H-0588 and 167.D-0407. a pulsator, e.g. the thickness of the outer hydrogen layer. Such results have been obtained, in the first application of asteroseismology to other stars than the sun, for the hotter WD pulsators of the PG1159 and DBV types. For the more common DAV type pulsators however, the comparatively small number of excited modes is a drawback. Kleinman (1999) has demonstrated that considering the combined pulsation modes of the whole DAV group instead of those of individual objects is an important step forward for DAV asteroseismology.

Besides being an important contribution to stellar astrophysics, such results facilitate the use of white dwarfs as tools in a number of different other research areas: cosmochronometry benefits from an improved knowledge of WD cooling rates, and in nuclear physics, WD asteroseismology can provide improved contraints on the ${ }^{12} \mathrm{C}(\alpha, \gamma){ }^{16} \mathrm{O}$ reaction rate. Furthermore, hDAV pulsators can be used to detect planetary companions of white dwarfs, because due to the high stability of hDAV oscillations ( $\dot{P} \simeq 4 \times 10^{-15} \mathrm{~s} \mathrm{~s}^{-1}$, Kepler et al. 2005a), an orbiting planet can be detected from variations of the observed arrival times of the pulses. For these reasons, a more substantial number of ZZ Cetis suitable for such projects, i.e. sufficiently bright objects with the right pulsational properties, would be an advantage. 
We have embarked on a program to identify a large number of bright new ZZ Ceti stars by conducting preparatory multicolour photometric observations of objects taken from Homeier $(2001)^{1}$, in order to determine the most promising candidate ZZ Ceti stars, and afterwards investigating their pulsations by time-series photometry. This paper presents the first results from this ongoing project.

In the last two years, there have been several new ZZ Ceti detections. Most of them, 60 in total, are objects of a rather faint magnitude that have been detected based on data of the Sloan Digital Sky Survey (SDSS) by Mukadam et al. (2004a), Mullally et al. (2005), and Kepler et al. (2005b). Today these 60 objects constitute the majority of known ZZ Ceti stars. Most recently, Silvotti et al. (2005) presented the discovery of two ZZ Ceti stars that had been selected as candidates by us, using the same selection methods as in this paper.

Here we report on the discovery of seven new ZZ Ceti stars. These stars were selected as candidates from the objects of Homeier (2001) and from the SPY sample (Napiwotzki et al. 2003). The total number of known ZZ Ceti stars, including those presented here, is now 105.

\section{Two-colour photometry}

The Hamburg Quasar Survey (HQS, Hagen et al. 1995) is an objective prism survey which, although primarily aimed at the discovery of extragalactic objects, is also a rich source of stellar objects of all kinds. For example, interesting results on pulsating sdB stars have been obtained from objects that were taken from this survey (e.g. Østensen et al. 2001a,b). Homeier (2001) derived a list of 3000 possible white dwarf stars from the lowresolution HQS spectra, including 1000 objects that are cool $\left(T_{\text {eff }} \lesssim 16000 \mathrm{~K}\right)$. Because of the large uncertainty of these temperatures, up to $\sim \pm 6000 \mathrm{~K}$, all of these are potential ZZ Ceti pulsators. As it is not feasible to obtain lightcurves of such a large number of objects, we conducted two-colour Strömgren observations at the Calar Alto $2.2 \mathrm{~m}$ telescope. These observations were designed to yield more precise temperatures and gravities for about 250 of the brightest $(B<16)$ stars in this sample.

We obtained photometry in the Strömgren $u, b, y$ and Johnson $I$ bands in four observation runs in between August, 2003, and March, 2005. We used the instrument BUSCA ${ }^{2}$, a four-channel CCD camera. The great advantage of this instrument is that four filters can be used simultaneously. Because the integrations through the different filters happen at the same time, the resulting colours are much less affected by varying atmospheric conditions than it would be the case if the integrations took place in the common sequential way.

The colours $u-b$ and $b-y$ show a strong dependency on $T_{\text {eff }}$ and $\log g$. This dependency is strongest in the region

\footnotetext{
1 Available in electronic form at http://www. astro.physik. uni-goettingen.de/ derek/lit/ diss_abstract.html

2 Bonn University Simultaneous Camera, see http://www.caha.es/guijarro/BUSCA/busca.html for further details.
}

of the ZZ Ceti instability strip. Therefore these two colours are well suited to select those objects that have the right temperature and gravity to be probable pulsators. However, in the course of our analysis, we noticed technical problems with the BUSCA $y$ filter. Furthermore, the temperature dependency of the more unusual colour $b-I$ is even stronger than that of $b-y$. Therefore we only use $u-b$ and $b-I$ data in our analysis, in spite of the fact that $b-I$ is a rather uncommon colour that can not be compared to any data in the literature. We do this analysis entirely in the BUSCA instrumental system, i.e. without transforming colours to the Strömgren standard system.

To obtain the colours, we use IRAF $^{3}$ aperture photometry, corrected for extinction. Then we compare these observed colours to synthetic colours that correspond to model atmospheres of known temperatures and gravities. These synthetic colours are computed by convolving model spectra with the transmission functions of the $u, b$, and $I$ filters through which the observations were made. The model spectra were computed from Koester DA model atmospheres. These are models of plane-parallel atmospheres in local thermodynamic equilibrium, in which convection is treated according to the mixing length approximation ML2/ $\alpha=0.6$. Following the notation of Fontaine et al. (1981), and Koester et al. (1994), "ML2" describes a certain combination of constants in the mixing length theory, and $\alpha$ is the mixing length in terms of the pressure scale height. Further details of the models are described e.g. in Finley et al. (1997).

This gives us a grid of synthetic photometry, which is however not on the BUSCA instrumental system and should require a transformation to that system. To derive this transformation, we also observed a number of known DA white dwarfs of different temperatures for which spectrophotometric data exists in the literature. We convolve these spectrophotometric literature data with the BUSCA filter curves, which gives us synthetic colours for these stars that are on the same system as the synthetic colours of the model atmospheres. The transformation itself is obtained by fitting the synthetic colours of each star to the observed colours of the respective star. We find a simple constant offset as the best transformation. After applying this offset, the observed colours, when plotted in a two-colour diagram of $u-b$ vs. $b-I$, are placed within the grid of synthetic colours that correspond to the model atmospheres of different $T_{\text {eff }}$ and $\log g$. This is displayed in Fig. 1, where we show 4 individual plots, one for each individual observation run.

Temperatures and gravities were determined by interpolating linearly between the grid points. By transforming the colour scatter of different observations of the same star to $T_{\text {eff }} / \log g$ space, we derive uncertainties of $\sim \pm 0.1$ in $\log g$ and $\sim \pm 300 \mathrm{~K}$ in $T_{\text {eff }}$. Compared to the original HQS data, this is an increase in accuracy by a factor of 20 . These $T_{\text {eff }}$ uncertainties are small compared to the width of the instability strip, thus allowing us to predict with a reasonable certainty whether an object is placed within or without the strip.

As a check of this method to select new ZZ Ceti variables, we included several known variables in our observations.

\footnotetext{
3 Image Reduction and Analysis Facility, see http://iraf.noao.edu
} 


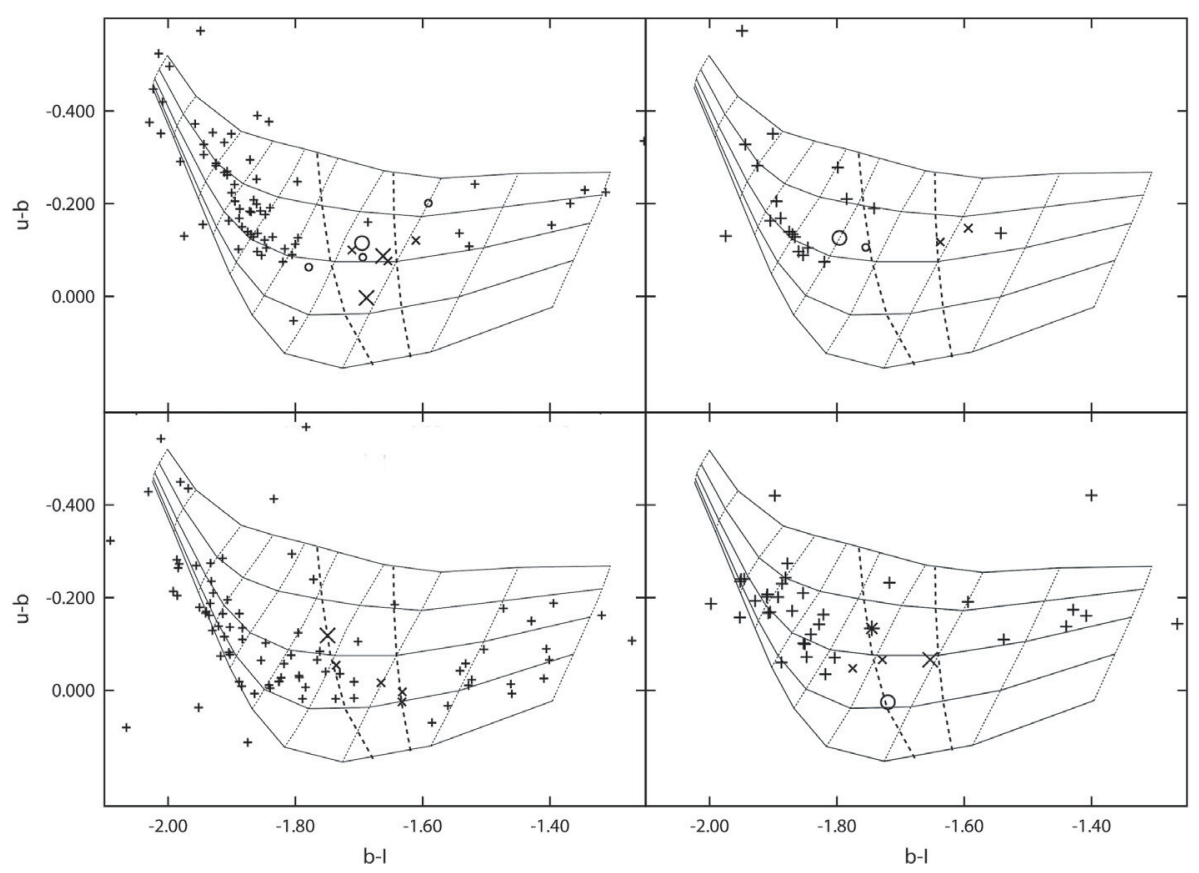

Fig. 1. The results of one of our two selection methods, two-colour photometry. Shown are the data of the different observation runs (2003/08, 2004/03, 2005/02, 2004/09, clockwise from upper left) and the grids of synthetic colours of model atmospheres. Solid lines of the grids are lines of constant gravity, from $\log g=7$ (bottom) to $\log g=9$ (top), and dotted lines are lines of constant temperature (from the left to the right: $20000 \mathrm{~K}, 18000 \mathrm{~K}, 16000 \mathrm{~K}$, and then down to $9000 \mathrm{~K}$ in steps of $1000 \mathrm{~K}$ ). Dashed lines show the empirical boundaries of the instability strip, according to Bergeron et al. (2004). The $\times$-symbols depict ZZ Ceti variables, and circles display targets for which the lightcurves show no sign of pulsations. +-Symbols denote objects whose variability status is unknown. The size of the symbols describes their relevance to this paper: the stars for which we obtained fast photometry that is presented here are shown as big $\times$-symbols and circles, while small $\times$-symbols and circles depict stars for which earlier lightcurve data is found in the literature. One object for which we obtained ambiguous results, HS 1441+3219, is shown as an asterisk. Some of these stars, especially some of those that are placed below the $\log g=7.0$ line, might not be white dwarf stars, as none of these objects has been spectroscopically identified as a white dwarf.

Considering Fig. 1, it can be noticed that most of these are indeed placed within the empirical boundaries of the instability strip, as defined by the empirical strip edges by Bergeron et al. (2004). Some variables are placed outside of the instability strip by our photometry, two of them by as much as $500 \mathrm{~K}$. Also in Fig. 1, a star for which no pulsations were detected is placed in the middle of the strip. Both, the placement of known variables outside the strip and the placement of stars without observed variations inside the strip, is largely explained if the temperature and $\log g$ uncertainties are taken into account. However it might indicate that the true uncertainties are somewhat larger than the above mentioned values. For such a conclusion however one would have to assume that the instability edges are precisely known, which is not the case. But even if the uncertainties in the photometrically estimated effective temperatures should be as large as $500 \mathrm{~K}$, the photometry would still allow a reasonably reliable selection of candidate ZZ Ceti stars because the uncertainties would still be as small as half the width of the instability strip.

From the data of the four observation runs, we derived temperatures and gravities for more than 200 stars, some of which are a few tenths of a magnitude fainter than the limiting magnitude of $B=16$ that had originally been planned for these observations. 26 of the observed objects are placed inside or very near to the instability strip and are thus possible new pulsators. We obtained time-series photometry for eight of these stars.
These stars are highlighted in Fig. 1, among them are four of the new variables. Three of the eight objects had been detected as white dwarfs earlier by other surveys; the remaining five are published here for the first time (see Table 1).

\section{Spectral analysis}

The remaining new variables where selected as candidates based on spectroscopy from the SPY (Supernova Ia Progenitor Survey) project (Napiwotzki et al. 2003), a radial velocity survey aimed at discovering double degenerate systems. The SPY produced high-resolution, high S/N spectra of approximately 700 DA WD, obtained at the ESO VLT UT2 telescope with the UVES spectrograph. Atmospheric parameters of these objects have been derived from the spectra by us, using the same model atmospheres that are used in the analysis of the two-colour photometry, and a fitting procedure that is described in Homeier et al. (1998). A paper on the results of this analysis is currently in preparation. Several SPY objects are placed inside the ZZ Ceti instability strip according to their effective temperatures, and in fact some earlier ZZ Ceti discoveries were partially based on preliminary SPY data (Fontaine et al. 2003; Bergeron et al. 2004).

SPY produced 2 independent spectra for most objects. Thus we are able to estimate the uncertainties of the fit results from a consideration of the individual fit results of two such 
Table 1. Properties of the observed ZZ Ceti candidate stars, according to the preparatory observations. Results from both spectral analysis and two-colour photometry are available for one object (WD 1149+057). For the objects with two-colour photometry, the last column indicates in which of the four runs the data was obtained.

\begin{tabular}{lllllllll}
\hline \hline Object & Alias & RA & $(\mathrm{J} 2000)$ & Dec & $B(\mathrm{mag})$ & $T_{\text {eff }}(\mathrm{K})$ & $\log g$ & method \\
\hline WD 1126-222 & EC 1126-2217 & 112911.6 & -223344 & 16.5 & 11989 & 7.83 & Spectroscopy \\
WD 1149+057 & PG 1149+058 & 115154.3 & +052838 & $15.1^{1}$ & 10978 & 8.06 & Spectroscopy \\
& & & & & 11105 & 7.95 & Photometry & $2005 / 02$ \\
HS 1249+0426 & PB 4304 & 125215.2 & +041043 & 15.8 & 11771 & 7.92 & Spectroscopy \\
WD 1300-098 & PG 1300-099 & 130316.8 & -100913 & $16.3^{1}$ & 14509 & 8.12 & Spectroscopy \\
WD 1342-237 & EC 13429-2342 & 134546.6 & -235711 & 16.1 & 10912 & 7.99 & Spectroscopy \\
HS 1441+3219 & CBS 391, WD 1441+323 & 144317.5 & +320657 & 16.4 & 12390 & 8.24 & Photometry & $2005 / 02$ \\
HS 1531+7436 & & 153035.4 & +742604 & 16.2 & 12357 & 8.17 & Photometry & $2004 / 09$ \\
HS 1544+3800 & & 154605.6 & +375127 & 14.4 & 13042 & 8.17 & Photometry & $2004 / 03$ \\
HS 1556+1634 & & 155840.3 & +162555 & 15.8 & 11439 & 7.55 & Photometry & $2005 / 02$ \\
HS 1612+5528 & SDSS 1612+555 & 161316.6 & +552126 & 15.9 & 11707 & 8.18 & Photometry & $2003 / 08$ \\
HS 1625+1231 & & 162813.8 & +122456 & 16.1 & 11272 & 8.06 & Photometry & $2003 / 08$ \\
HS 1824+6000 & & 182444.3 & +600158 & 15.7 & 11192 & 7.65 & Photometry & $2003 / 08$ \\
\hline
\end{tabular}

${ }^{1}$ PG photographic magnitude (Green et al. 1986).

corresponding spectra. We adopt the mean difference of such pairs of two $T_{\text {eff }}$ and $\log g$ values as the uncertainties of all fit results, and find uncertainties of 0.048 dex for $\log g$ and of $181 \mathrm{~K}$ for $T_{\mathrm{eff}}$.

We find eleven ZZ Ceti candidates in the SPY sample, and five of these were observed with high-speed photometry. We show the SPY spectra and the fits of these five objects in Fig. 2. Four of the five objects turned out to be variable. One of these spectroscopically selected candidates has also been selected as a candidate from the two-colour photometry. The results from both preparatory methods, spectral fitting and two-colour photometry analysis, are listed in Table 1 and are displayed, together with the fast photometry results, in Fig. 7.

\section{Time series observations and reduction}

The time series observations were carried out in five nights from 2005-05-13 to 2005-05-18 at the $2.5 \mathrm{~m}$ Nordic Optical Telescope (NOT) at the Roque de los Muchachos Observatory, Spain, and on 2005-06-08 at the $4.1 \mathrm{~m}$ Southern Observatory for Astrophysical Research (SOAR) telescope on the Cerro Pachon, Chile.

The NOT observations were done with $\mathrm{ALFOSC}^{4}$, using an E2V back-illuminated $2048 \times 2048$ chip. This instrument is equipped with a controller and a controller software that allow us to read out the chip in a multi-window mode. Thereby it is possible to achieve the short cycle times that are needed to resolve the highest frequency ZZ Ceti pulsations. A typical windowed readout takes about $5 \mathrm{~s}$, compared to $90 \mathrm{~s}$ for a full frame. The employed window size was $64 \times 64$ pixels, corresponding to about $12^{\prime \prime}$ on the sky, and the observations were made through a sky contrast filter (NOT \# 92) with a bandwidth of $2750 \AA$, centered on $5500 \AA$.

\footnotetext{
${ }^{4}$ Andalucia Faint Object Spectrograph and Camera, see http://www.not.iac.es/instruments/alfosc/ for more details on the instrument, and Østensen \& Solheim (2000) for information about the fast photometry controller and software.
}

The integration times were between $9.9 \mathrm{~s}$ and $50 \mathrm{~s}$, leading to cycle times between $15 \mathrm{~s}$ and $56 \mathrm{~s}$, depending on the target magnitude. In addition to the target star window, at least five additional windows were read from the CCD, at least three of them centered on the best comparison stars available in the field and two on empty sky fields. More details about sequence lengths and cycle times are given in the NOT observation journal in Table 2.

Data reduction was done by a combination of IRAF and RTP (Østensen \& Solheim 2000) $)^{5}$. After flat fielding and sky subtraction, weighted differential aperture photometry was carried out with a fixed aperture, optimized for the highest $\mathrm{S} / \mathrm{N}$ in the fourier transform (FT) of the resulting lightcurve. The final lightcurves were corrected for any linear trend.

At the SOAR telescope, we used the SOAR Optical Imager, a mosaic of two E2V $2048 \times 4096$ CCDs, thinned and back illuminated, with an efficiency around $73 \%$ at $4000 \AA$, to acquire time series photometry. It covers a field of $5.26^{\prime} \times 5.26^{\prime}$ on the sky, on the bent cassegrain port of the telescope. We observed in fast readout mode, with the CCDs binned $4 \times 4$, which resulted in a pixel scale of 0.308 arcsec/pixel and a readout and write time of $6 \mathrm{~s}$. The observations started at 23:32:23.1 UT on 2005-06-08. The exposure times were $20 \mathrm{~s}$. The data was bias subtracted and flat fielded before we obtained differential photometry through weighted apertures with a radius of 2 FWHM (full width at half maximum of the seeing disk), chosen for highest SNR.

\section{The new variables}

Because conditions were partially clouded throughout the NOT run, only 12 objects could be observed, which is less than half of the planned number. For the same reason, the lightcurves of some objects are rather short and thus the resulting frequency resolutions are rather low. For two of the new

\footnotetext{
5 Also see http://www.not.iac.es/instruments/alfosc/ fastphot/.
} 


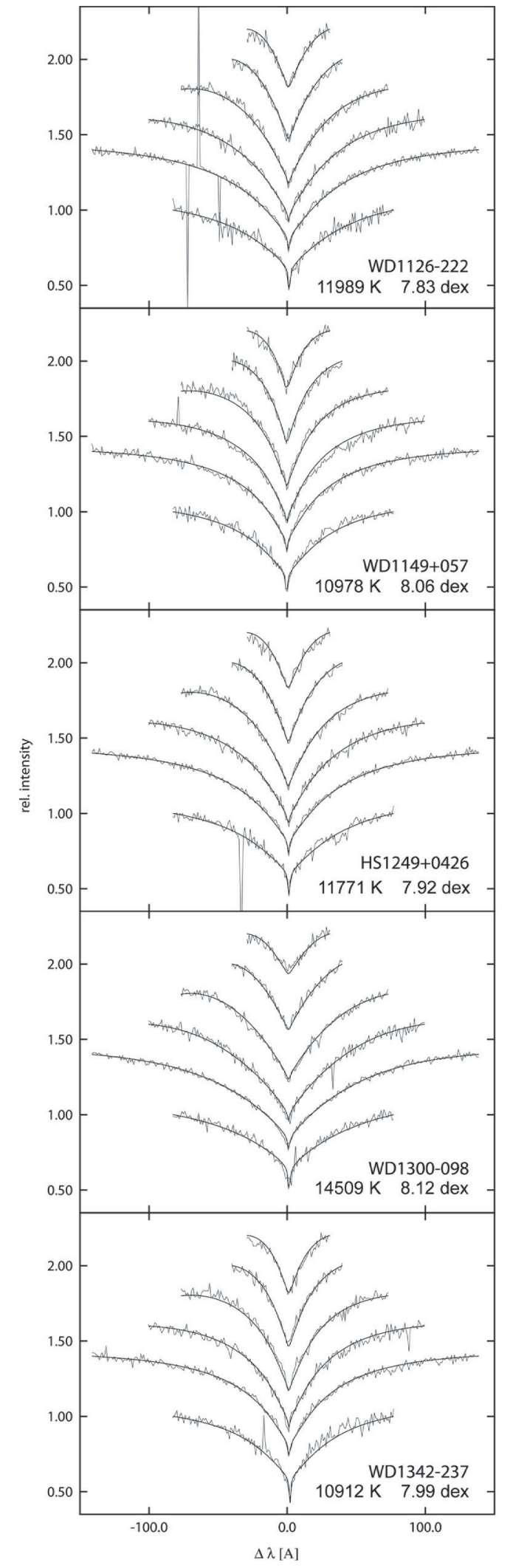

Fig. 2. The SPY spectra and DA model atmosphere fits. The spectra were rebinned to a resolution of $1 \AA$ for this plot, but the fit was done with the original resolution of $0.1 \AA$. The Balmer lines are plotted in a stacked fashion, with $\mathrm{H} \alpha$ at the bottom and $\mathrm{H} 8$ on top. The cosmics that are visible in some of the spectra do not affect the fitting process significantly. The values of $T_{\text {eff }}$ and $\log g$ that were derived from these fits are given in each plot. variables, HS 1249+0426 and WD 1149+057, only a very short lightcurve barely covering one pulsation cycle could be obtained. However, the variability is unambiguously present even in these cases. For three of the new variables, data from more than one night exists. These lightcurves are, due to the cloudy conditions, mostly rather short and are separated by several days. Therefore a combined FT produces alias patterns that prevent any effective gain of the frequency resolution, because the alias pattern is so severe that it can not be determined which of the several peaks is the strongest peak that would correspond to the target's pulsation frequency. Thus, a combined FT of all data of one object does not provide an advantage compared to the FT of a single data set, and so we only show the separate lightcurves and the corresponding separate FTs in Fig. 5. The pulsation frequencies and amplitudes that we derive from the FTs are listed in Table 4. The noise in each FT was determined by computing $\langle A\rangle$, the square root of the average power, which is an estimate of the noise (Schwarzenberg-Czerny 1991, 1999; Kepler 1993). Ideally this would be done locally, and not for the entire FT all at once, but our FTs do not have a resolution that would be sufficient to derive such a value of the noise with respect to the frequency. Therefore we only determine the overall noise and for that we use wide regions in the FT that cover most of the spectrum, only avoiding the very low frequencies and the peaks in the FT. If clouds are present during the observation, they often induce low-frequency variations. These are also seen in the differential lightcurves, because the colours of the blue white dwarf target star, and the comparison stars which are average field stars, and thus rather red, are very different, which causes noticeable differential extinction. This can lead to an increased noise in the affected frequency region, normally below $2 \mathrm{mHz}$, and thus an overall estimate of the noise is not useful in such cases in this region of the FT. Therefore, the low-frequency FT peaks of HS 1625+1231 and WD 1149+057 might be somewhat less significant than listed in Table 4.

Two of the new variables, WD 1126-222 and HS 1625+ 1231, show multiple periods; and in the case of WD 1126-222 there is evidence for a variation of the amplitudes in between different nights. HS 1824+6000 also shows some differences in the measured amplitudes, but the runs are too short to judge whether this is a real effect, or just a consequence of beating between closely spaced modes. Three of the seven new ZZ Cetis are long-period pulsators (WD 1149+057, WD 1342-237, and HS 1625+1231), the remaining four have periods below $400 \mathrm{~s}$. Among these is HS $1531+7436$ which shows a period of only 112 s, i.e. one of the shortest periods of ZZ Cetis known to date.

In addition to the seven new variables, there are five observed candidates for which no clear variations were detected. One has to be careful about classifying these objects as nonvariables, as it is quite possible that weak variations are present below the noise level of the present observations. Even more importantly, strong variations may be present but temporarily hidden from view due to beating effects that are known to cause almost flat lightcurves for over one hour in many ZZ Ceti stars. Therefore it is more meaningful to refer to these objects as "not observed to vary" (NOV) than as "Non-Variables". The level at which variations can be excluded in the individual lightcurves is given in Table 3 . 
Table 2. Journal of observations with the NOT, ordered by starting date $\&$ time.

\begin{tabular}{lllrrrr}
\hline \hline Object & Date & $\begin{array}{l}\text { Starting time } \\
\text { (UTC) }\end{array}$ & \multicolumn{2}{r}{$\begin{array}{r}\text { Integration and cycle } \\
\text { times (s) }\end{array}$} & $\begin{array}{r}\text { Length } \\
(\mathrm{s})\end{array}$ & $\begin{array}{l}\text { Observing } \\
\text { conditions }\end{array}$ \\
\hline WD 1126-222 & $2005-05-13$ & $21: 07: 20$ & 25.1 & 30 & 3630 & \\
WD 1300-098 & $2005-05-13$ & $22: 51: 24$ & 29.1 & 30 & 1830 & \\
WD 1342-237 & $2005-05-13$ & $23: 35: 22$ & 26.1 & 30 & 1950 & interrupted by clouds \\
HS 1544+3800 & $2005-05-14$ & $00: 34: 34$ & 9.9 & 15 & 1275 & \\
HS 1531+7436 & $2005-05-14$ & $01: 08: 10$ & 33.3 & 40 & 3800 & \\
HS 1612+5528 & $2005-05-14$ & $02: 59: 55$ & 24.1 & 30 & 2280 & \\
HS 1824+6000 & $2005-05-14$ & $03: 47: 07$ & 22.7 & 30 & 1290 & interrupted because of ToO observations \\
HS 1441+3219 & $2005-05-15$ & $00: 21: 42$ & 38.5 & 45 & 1620 & interrupted by clouds \\
HS 1625+1231 & $2005-05-15$ & $01: 00: 04$ & 28.5 & 35 & 5005 & \\
HS 1824+6000 & $2005-05-15$ & $03: 03: 11$ & 22.6 & 30 & 2400 & interrupted by clouds \\
WD 1126-222 & $2005-05-16$ & $21: 43: 44$ & 50.0 & 56 & 2432 & interrupted by clouds \\
WD 1149+057 & $2005-05-16$ & $23: 25: 41$ & 35.1 & 40 & 2480 & interrupted by clouds \\
HS 1249+0426 & $2005-05-18$ & $01: 12: 20$ & 40.0 & 45 & 1890 & interrupted by clouds \\
HS 1441+3219 & $2005-05-18$ & $02: 40: 18$ & 38.1 & 45 & 1845 & \\
HS 1556+1634 & $2005-05-18$ & $03: 20: 28$ & 29.5 & 35 & 2555 & \\
HS 1531+7436 & $2005-05-18$ & $04: 10: 30$ & 23.4 & 30 & 1710 & \\
HS 1824+6000 & $2005-05-18$ & $04: 47: 31$ & 22.4 & 30 & 1170 & \\
\hline
\end{tabular}

Table 3. Objects not observed to vary. Given are the square root of the average power in the FT of the object's lightcurve $(\langle A\rangle)$, and the amplitude of the highest peak in the FT. All of the peaks are close to $2 \sigma$ in these observations, which we consider as insignificant.

\begin{tabular}{lll}
\hline \hline Object & $\langle A\rangle(\mathrm{mma})$ & Highest peak $(\mathrm{mma})$ \\
\hline WD 1300-098 & 0.60 & 1.09 \\
HS 1441+3219 & 0.91 & 2.02 \\
HS 1544+3800 & 0.38 & 0.70 \\
HS 1556+1634 & 0.51 & 0.94 \\
HS 1612+5528 & 0.66 & 1.33 \\
\hline
\end{tabular}

\subsection{WD 1126-222}

Two lightcurves were obtained for WD 1126-222, the first one being much longer than the second and thus providing a higher frequency resolution. The long-period peak at $0.5 \mathrm{mHz}$ is not real but due to a long-term trend that was not properly removed by the linear detrending of the data. This residual trend is probably due to differential extinction by clouds, since the colours of the reference stars are typically much redder than that of the white dwarf target star.

The amplitude of one of the peaks in the FT, at $4.6 \mathrm{mHz}$, increased by a factor of 2.2 in between the runs, while the other major peak at $3.6 \mathrm{mHz}$ is of almost constant strength.

\subsection{WD 1149+057 and HS $1249+0426$}

Only scarce NOT data exists for these new ZZ Cetis, again due to the unfavourable weather conditions. In spite of the very short lightcurves and rather inaccurate determination of their pulsation frequencies, these objects are clearly variable. Further observations are however needed to derive precise pulsational parameters. Only the main peak in the FT
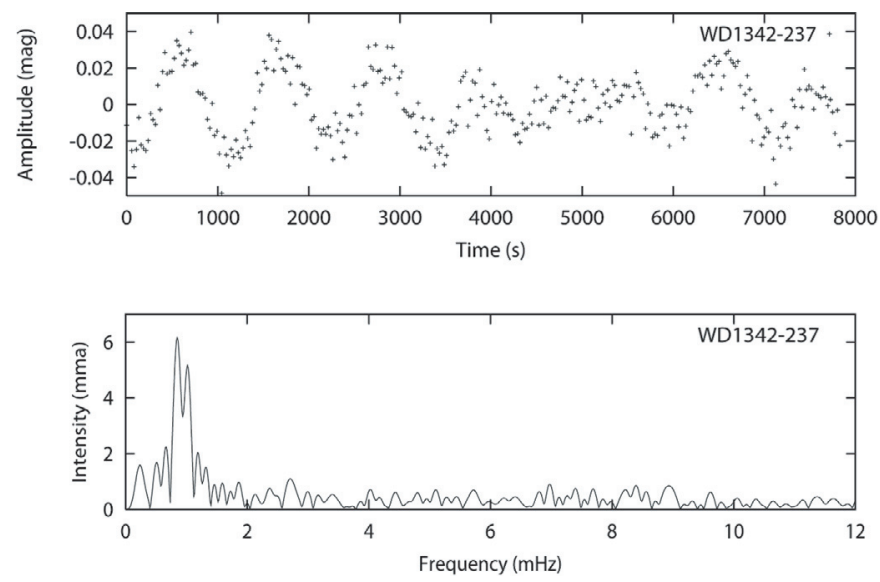

Fig. 3. SOAR photometry of WD 1342-237 (above) and the fourier transform of the lightcurve (below).

of HS $1249+0426$ is significant, all other features in that FT are not.

WD $1149+057$ has a very long period, and this agrees well with the result of the spectral analysis that places it on the cool edge of the instability strip.

\subsection{WD 1342-237}

As in the case of WD 1159+057 and HS 1249+0426, the NOT lightcurve of this object is short and covers only one pulsational cycle. These data do not allow a derivation of precise parameters for this object, but we also have obtained fast photometry of WD 1342-237 at the SOAR telescope. This lightcurve is of much higher quality. It is displayed along with its FT in Fig. 3; in Table 4 we only list the frequencies and amplitudes of WD 1342-237 that have been derived from these SOAR data and not those derived from the NOT lightcurve. The high-resolution data allows us to resolve the main peak into 
Table 4. Observed periods and amplitudes. The given resolution is the inverse of the length of the run. The last column gives the significance of the individual peaks, in $\sigma$ levels above the noise, which was determined in featureless regions of each FT. Uncertain data are emphasized; these include the significances of the low-frequency peaks of WD 1149+057 and HS 1625+1231 as well as some peaks in the FT of HS 1625+1231 that are probably caused by cloud interference or are probable alias peaks.

\begin{tabular}{|c|c|c|c|c|c|c|}
\hline Object & Date & $\begin{array}{r}\text { Resolution } \\
(\mathrm{mHz})\end{array}$ & $\begin{array}{r}\text { Frequency } \\
\qquad(\mathrm{mHz})\end{array}$ & $\begin{array}{r}\text { Amplitude } \\
\text { (mma) }\end{array}$ & $\begin{array}{r}\text { Period } \\
\text { (s) }\end{array}$ & $\begin{array}{r}\text { Significance } \\
(\sigma)\end{array}$ \\
\hline \multirow[t]{7}{*}{ WD 1126-222 } & 2005-05-13 & 0.28 & 2.48 & 3.01 & 402.7 & 5.5 \\
\hline & & & 3.60 & 7.04 & 277.6 & 12.8 \\
\hline & & & 4.27 & 4.49 & 234.1 & 8.2 \\
\hline & & & 4.64 & 3.68 & 215.7 & 6.7 \\
\hline & 2005-05-16 & 0.41 & 2.59 & 4.01 & 386.4 & 4.5 \\
\hline & & & 3.63 & 7.69 & 275.7 & 8.5 \\
\hline & & & 4.58 & 8.13 & 218.3 & 9.0 \\
\hline WD $1149+057$ & 2005-05-16 & 0.40 & 0.98 & 10.47 & 1023.5 & 7.7 \\
\hline HS $1249+0426$ & 2005-05-18 & 0.53 & 3.46 & 7.55 & 288.9 & 6.6 \\
\hline \multirow[t]{2}{*}{ WD 1342-237 } & 2005-06-08 & 0.13 & 0.85 & 6.17 & 1177.0 & 16.2 \\
\hline & & & 1.02 & 5.17 & 982.0 & 13.6 \\
\hline \multirow[t]{2}{*}{ HS $1531+7436$} & 2005-05-14 & 0.26 & 8.89 & 4.23 & 112.5 & 8.1 \\
\hline & 2005-05-18 & 0.59 & 8.97 & 3.38 & 111.5 & 4.4 \\
\hline \multirow[t]{10}{*}{ HS $1625+1231$} & 2005-05-15 & 0.20 & 0.43 & 14.27 & 2309.8 & 25.9 \\
\hline & & & 1.16 & 48.87 & 862.9 & 88.9 \\
\hline & & & 1.87 & 23.60 & 533.6 & 42.9 \\
\hline & & & 2.35 & 13.90 & 425.8 & 25.3 \\
\hline & & & 2.60 & 16.99 & 385.2 & 30.9 \\
\hline & & & 2.83 & 10.69 & 353.0 & 19.4 \\
\hline & & & 3.07 & 13.31 & 325.5 & 24.2 \\
\hline & & & 3.73 & 13.34 & 268.2 & 24.3 \\
\hline & & & 4.02 & 9.87 & 248.9 & 17.9 \\
\hline & & & 4.91 & 7.77 & 203.7 & 14.1 \\
\hline \multirow[t]{4}{*}{ HS $1824+6000$} & 2005-05-14 & 0.78 & 3.40 & 8.84 & 294.3 & 8.9 \\
\hline & 2005-05-15 & 0.42 & 2.60 & 3.30 & 384.4 & 5.9 \\
\hline & & & 3.29 & 7.66 & 304.4 & 13.7 \\
\hline & $2005-05-18$ & 0.86 & 3.03 & 13.56 & 329.6 & 19.3 \\
\hline
\end{tabular}

two components that are separated by $0.17 \mathrm{mHz}$. According to the spectral analysis, this object should be a cDAV, which is consistent with the long periods of its pulsations.

\subsection{HS $1441+3219$}

This object shows a peak at $5.6 \mathrm{mHz}$ which seems fairly pronounced. We consider this peak as an indication of uncertain possible variations of HS $1441+3219$. This feature is only found in the FT of the second of two lightcurves, but its absence in the FT of the first lightcurve is easily explained by the much higher noise of more than $3 \mathrm{mma}$ in that first FT. The square root of the average power in the second FT is $0.91 \mathrm{mma}$, so the peak, which has an amplitude of $2.02 \mathrm{mma}$, is in fact below the significance level. Therefore it is included in the list of NOV objects, Table 3. Although there is no evidence for pulsations of HS $1441+3219$, we suspect that it may be variable, and plan to do follow-up observations of this candidate.

\subsection{HS 1531+7436}

Two lightcurves were obtained for HS $1531+7436$, but only the first one (2005-05-14) is of good quality. The long-period peak at $0.3 \mathrm{mHz}$ in the FT of the first lightcurve is, like that in the FT of WD 1126-222, probably due to a long-term atmospheric trend in the data that was not properly removed by a linear correction. According to the two-colour photometry, this object should be an extreme hDAV, which is indeed consistent with the exceptionally short period of only $112 \mathrm{~s}$.

\subsection{HS $1625+1231$}

Five consecutive points of the lightcurve of HS 1625+1231 are outliers with respect to the otherwise undisturbed lightcurve. These points have been rejected and a linear interpolation was used to replace them. 


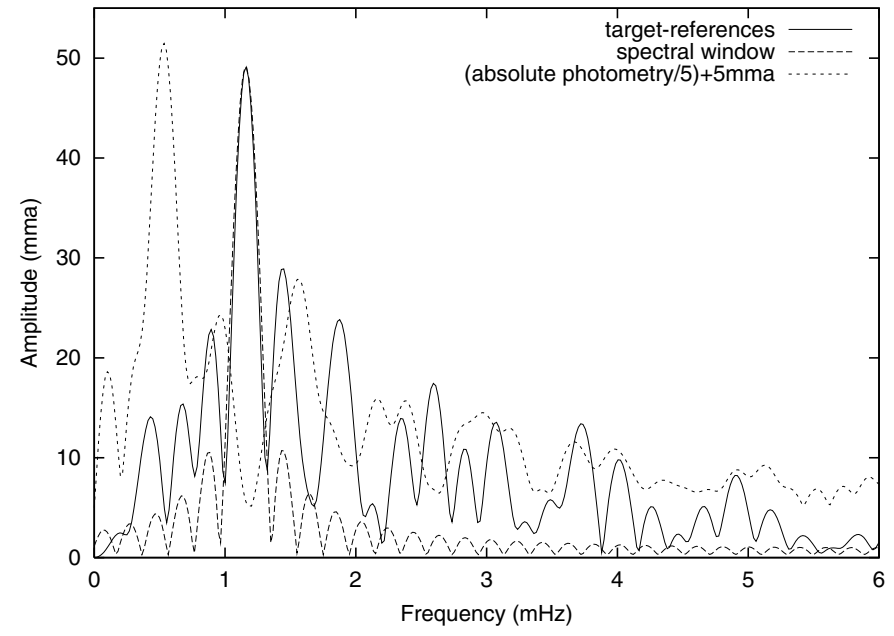

Fig. 4. Comparison of the FT of the differential lightcurve of HS $1625+1231$ to the spectral window, and to the FT of the absolute (non-differential) photometry of a reference star. The absolute photometry shows atmospheric variations. It has been scaled to a fifth of its amplitude and shifted up by $5 \mathrm{mma}$ for better visibility in this plot. The spectral window curve has been scaled to match the peak amplitude of the main peak in the FT. One can see that many of the peaks in the FT of HS $1625+1231$ are due to aliases or to variations that are also present in the absolute FT, and thus are probably not real features but caused by cloud interference.

The FT shows a high number of at least 13 seemingly significant peaks. If the FT is compared to the spectral window, as shown in Fig. 4, one finds that the peaks at 0.7, 0.9, 1.4, and possibly also that at $0.4 \mathrm{mHz}$ are probably alias peaks, as they coincide with peaks in the spectral window. Still, these peaks would be strangely high above the noise level.

As mentioned earlier in this section, that can be explained by the fact that clouds were disturbing the observation of this lightcurve, causing low-frequency variations which also affect the differential lightcurves and increase the low-frequency noise. Therefore, in the case of this object, the true noise at low frequencies has to be higher than the overall estimate, and thus the above mentioned peaks are probably insignificant.

Furthermore, we find that most peaks in the differential FT correspond to features in the FT of the absolute photometry of the reference stars, which is also shown in Fig. 4. We suspect that these coincidential peaks as well are reflections of cloud interference. The only peaks that we expect to be real pulsation features of the target star are, besides the main peak, those at 1.8 and possibly that at $2.7 \mathrm{mHz}$.

\subsection{HS $1824+6000$}

Data of HS $1824+6000$ has been obtained on three nights, although two of the three lightcurves are too brief to yield meaningful frequencies. The shift of the frequency of the main peak, especially from the second to the third lightcurve, is large but less than a third of the peak width in the 2005-05-18 FT. Thus this shift is not significant. Also, the length of the runs is too short to be able to regard the change in amplitude as a real change.

\section{Results and discussion}

We have obtained high-speed photometry of twelve objects that were taken either from Homeier (2001), or from the SPY sample. We selected these stars as candidate ZZ Ceti stars by deriving their effective temperatures and gravities from a spectral analysis and from an analysis of two-colour photometry data, respectively. Seven of the twelve observed targets turned out to be pulsating and are thus new ZZ Ceti stars. Finding charts for these objects are provided in Fig. 6.

The ratio of candidates that were selected from spectroscopy to the number of variables among these candidates is relatively high, 0.8 , similar to values that have been found by other studies (e.g. Mukadam 2004a). This number is not much lower for the photometrically derived candidates. Six of these are placed inside or very close to the instability strip, of which 4 are found to be variable, leading to a "success fraction" of 0.66 for this method.

Compared to the typical magnitude of the numerous ZZ Ceti stars from the SDSS $(B \approx 18)$ that have recently been published, the objects described here are relatively bright, with $B \approx 16$, which makes them more suitable candidates for detailed asteroseismological analyses.

In Fig. 7 the results of the observations are plotted in the $T_{\text {eff }} / \log g$-plane. We also plot the empirical instability strip edges by Bergeron et al. (2004) and Mukadam et al. (2004b). The Bergeron boundaries are a better match to our atmospheric parameters of the new variables than the Mukadam edges. This is mainly due to the Mukadam blue edge, which seems too red at high values of $\log g$. However, considering Fig. 5 in Mukadam et al. (2004b), one can see that their blue edge is not well constrained by their observations at high values of $\log g$. It is possible that it might turn out to be more similar to the Bergeron edge if objects with higher values of $\log g$ and $T_{\text {eff }}$ would be included in its determination. The ZZ Ceti stars that we find in this region of the $T_{\text {eff }} / \log g$-diagram, HS $1531+7436$ and possibly HS $1441+3219$, might therefore prove valuable for further investigations of the blue edge of the ZZ Ceti instability strip.

We cannot make any firm statement on the purity of the instability strip, as the uncertainties of the two-colour photometry might be as big as half the width of the instability strip (see Sect. 2). The object HS $1612+5528$, which is placed in the middle of the strip but shows no variations might therefore have a true effective temperature that in fact places it outside of the strip. On the other hand, because our lightcurve of that target is not very long, it is also well possible that this object is indeed a variable, with pulsations that were temporarily hidden due to beating effects. This possibility is supported by the fact that HS $1612+5528$, of all of our NOV objects, has the highest noise in the FT and is therefore the least constrained of our NOV stars.

Therefore, and also because the number of NOV objects that we found is too small, we can merely say that our results are consistent with a pure instability strip.

Another conclusion is that the pulsational properties of the new variables are in very good agreement with the atmospheric parameters that we determined: the stars for which we 

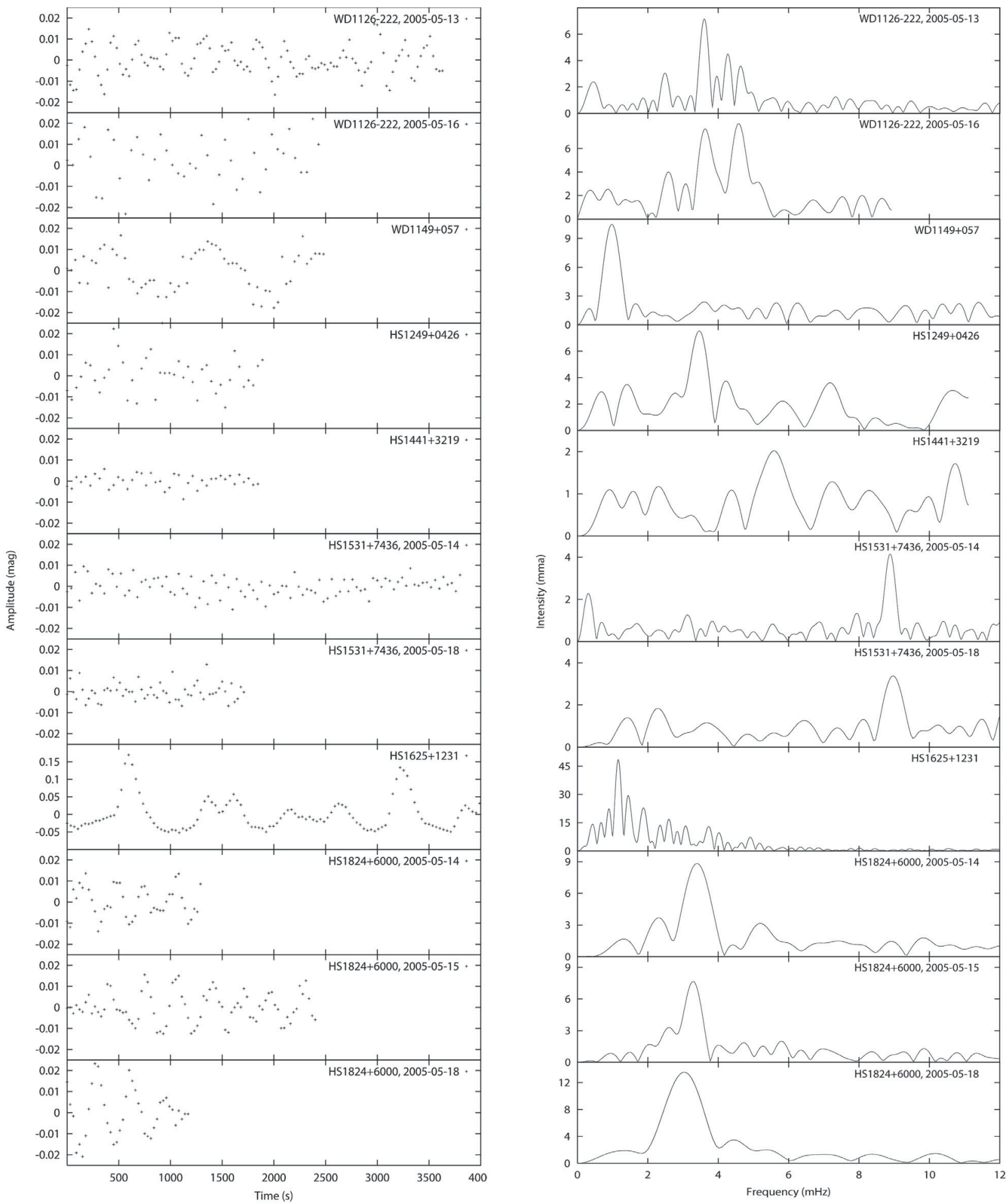

Fig. 5. Differential NOT photometry (left) and the fourier transforms of the lightcurves (right) of the new ZZ Ceti variables and of the ambiguous object HS 1441+3219. For the targets for which data was obtained on more than one night, these individual lightcurves are shown separately. Note the scaling of the intensity axes, which is not the same for all objects.

derive cDAV atmospheric paramaters show long periods, and vice versa. This adds some additional confidence to our atmospheric parameters, and especially to the accuracy of the photometrically derived ones.

For several of the ZZ Ceti candidates that have been identified in the course of this project (see Sects. 2 and 3), no lightcurves have yet been obtained, because of unfavourable weather conditions throughout the NOT run in May 2005 and, as the targets are distributed equally across the sky, because not all can be observed in one run. We are therefore planning further lightcurve observations, mainly of the unobserved ZZ Ceti candidates, but also as a follow-up on some of the objects that 


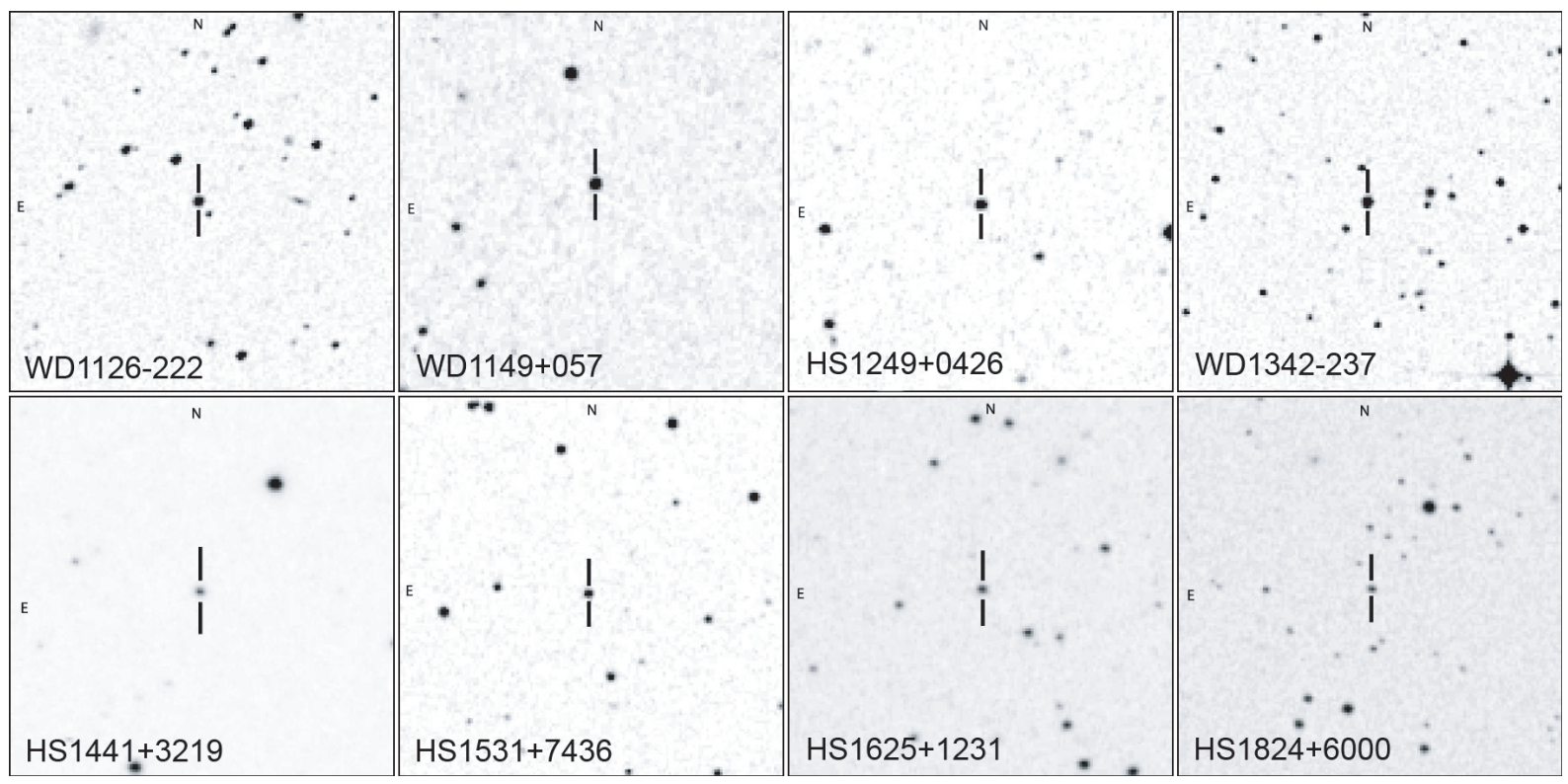

Fig. 6. Finding charts for the seven new variables, and for HS $1441+3219$, which is an ambiguous object that shows some signs of possible variability. In all charts, the scale is $4^{\prime} \times 4^{\prime}$, with north on top, and east to the left.

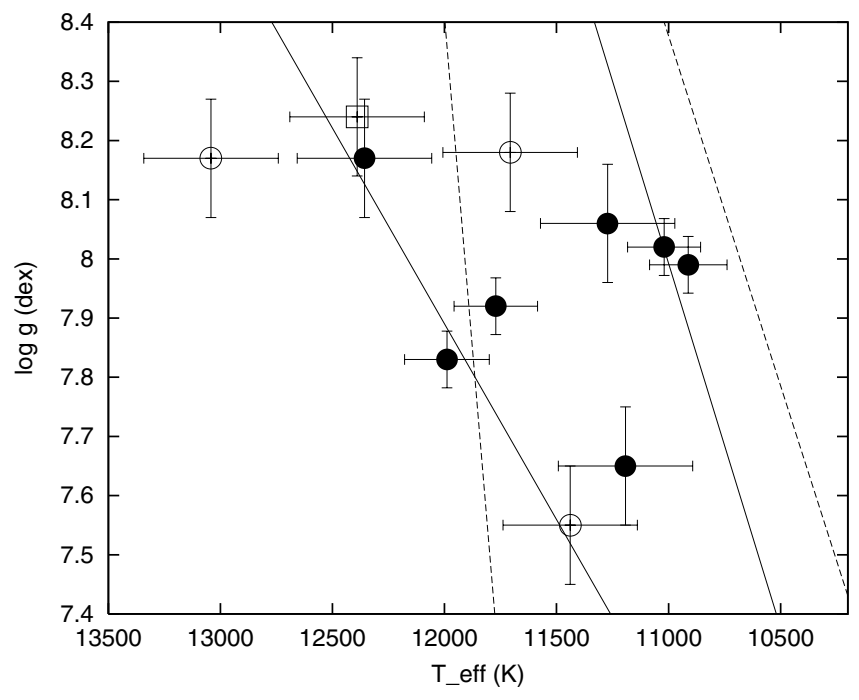

Fig. 7. The results of the atmospheric parameter determination and of the time-series observations, plotted in the $T_{\text {eff }}$ vs. $\log g$ plane. The seven new variables are shown as filled circles, and the NOV objects as open symbols. HS 1441+3219, which counts among the non-variables according to our data but might in fact show weak variations is displayed as a square symbol. The fifth NOV object is placed outside of the diagram. The instability strip boundaries according to Bergeron et al. (2004) are plotted as solid lines; those from Mukadam et al. (2004b) are shown as dashed lines.

we report on here. By multiplying the number of unobserved ZZ Ceti candidates with the above mentioned "success fractions", we arrive at an estimated number of more than 10 additional new $\mathrm{ZZ}$ Ceti stars that should be detected from our remaining candidates.

Acknowledgements. B.V. and D.K. acknowledge support by the Deutsche Forschungsgemeinschaft (DFG), under project numbers KO738/21-1, KO738/22-1, KO738/23-1, and by a travel grant from the OPTICON Trans-national access programme. R.N. acknowledges support by a PPARC Advanced Fellowship.

\section{References}

Bergeron, P., Fontaine, G., Billéres, M., Boudreault, S., \& Green, E. M. 2004, ApJ, 600, 404

Finley, D. S., Koester, D., \& Basri, G. 1997, ApJ, 487, 375

Fontaine, G., Villeneuve, B., \& Wilson, J. 1981, ApJ, 243, 550

Fontaine, G., Bergeron, P., Billéres, M., \& Charpinet, S. 2003, ApJ, 591,1184

Green, R. F., Schmidt, M., \& Liebert, J. 1986, ApJS, 61, 305

Hagen, H.-J., Groote, D., Engels, D., \& Reimers, D. 1995, A\&AS, 111,195

Homeier, D. 2001, Ph.D. Thesis, University Kiel

Homeier, D., Koester, D., Hagen, H.-J., et al. 1998, A\&A, 338, 563

Kepler, S. O. 1993, Baltic Astron., 2, 515

Kepler, S. O., Robinson, E. L., Koester, D., et al. 2000, ApJ, 539, 379

Kepler, S. O., Castanheira, B. G., Saraiva, M. F. O., et al. 2005, A\&A, 442,629

Kepler, S. O., Costa, J. E. S., Castanheira, B. G., et al. 2005, AJ, 634, 1311

Kleinman, S. J. 1999, in 11th European Workshop on White Dwarfs, ed. J.-E. Solheim, \& E. G. Meistas, ASP Conf. Ser., 169, 116

Koester, D., Allard, N., \& Vauclair, G. 1994, A\&A, 291, L9

McCook, G. P., \& Sion, E. M. 1999, ApJS, 121, 1

Mukadam, A. S., Mullally, F., Nather, R. E., et al. 2004a, ApJ, 607, 982

Mukadam, A. S., Winget, D. E., von Hippel, T., et al. 2004b, ApJ, 612, 1052

Mullally, F., Thompson, S. E., Castanheira, B. G., et al. 2005, ApJ, 625,966

Napiwotzki, R., Christlieb, N., Drechsel, H., et al. 2003, Msngr, 112, 25

Østensen, R., \& Solheim, J.-E. 2000, BaltA, 9, 411

Østensen, R., Solheim, J.-E., Heber, U., et al. 2001a, A\&A, 368, 175

Østensen, R., Heber, U., Silvotti, R., et al. 2001b, A\&A, 378, 466

Schwarzenberg-Czerny, A. 1991, MNRAS, 253, 198

Schwarzenberg-Czerny, A. 1999, ApJ, 516, 315

Silvotti, R., Voss, B., Bruni, I., et al. 2005, A\&A, 443, 195 